\title{
RESPONSE OF CRESTED AND NONCRESTED DANDARAWI CHICKENS TO EXCESS L-ARGININE SUPPLEMENTATION DURING WINTER MONTHS.
}

\author{
S. F. Youssef; H. A. H. Abd El-Halim. and M. I. Badawy \\ Anim. Prod. Res. Inst. Agric. Res. Center, Minis. of Agric., Dokki, Giza, Egypt.
}

Corresponding author: sabbah.farouk@yahoo.com

Received: 10/08/2017

Accepted: 29/08/2017

\begin{abstract}
The present study was carried out during winter months to estimate response of two Dandarawi chicken phenotypes (crested $(\mathrm{Cr})$ and non-crested (cr)) to extra L-arginine (Arg) supplementation. Seventy two female from each of $\mathrm{Cr}$ and cr layer Dandarawi hens were chosen when egg production(EP) reached $50 \%$. The $\mathrm{Cr}$ and cr layer Dandarawi hens were distributed randomly into 3 experimental groups; each group includes 24 layer hens divided randomly into 3 replicates with 8 hens each. The $1^{\text {st }}$ group from each phenotype fed control basal diet that satisfied recommendations of NRC. Groups 2 and 3 from each type fed basal diet supplemented with $2 \%$ and $4 \%$ L- arginine over NRC requirements respectively for three winter months. Arginine supplemented by $14 \mathrm{gm} / 100 \mathrm{~kg}$ diet and $28 \mathrm{gm} / 100 \mathrm{~kg}$ to perform levels $2 \%$ and $4 \%$ respectively.

The following results were obtained:-

Crested phenotype had higher significantly EP\% and egg mass per hen per day than cr during $2^{\text {nd }}$ month and overall period moreover $\mathrm{Cr}$ laying hen consumed significantly lower diets than cr during the first month of EP and improved significantly feed conversion during the second month of EP. Significant decrease was observed in yolk percent of egg laid by $\mathrm{Cr}$ hens but significant increase was observed in shell thickness. Body temperature of $\mathrm{Cr}$ phenotype during chronic cold condition was lower by about $0.38^{\circ} \mathrm{C}$ than $\mathrm{cr}$ phenotype and had low significantly respiration rate.

Diets supplemented with excess Arg recorded significantly $(\mathrm{P} \leq 0.05)$ higher EP\% than control diet during $2^{\text {nd }}$ month, $3^{\text {rd }}$ month and for overall period. Excess Arg supplementation reduced significantly feed intake during the $1^{\text {st }}$ month of EP, but increased significantly feed intake during the $3^{\text {rd }}$ month. Increasing Arg level to $4 \%$ increased significantly $(\mathrm{P} \leq 0.05)$ yolk percent and decreased significantly $(\mathrm{P} \leq 0.05)$ albumin percent.
\end{abstract}

Key Words: Crest, Dandarawi, arginine, cold stress and egg production. 


\section{INTRODUCTION}

Overcoming cold stress is one of the most important challenges in poultry production. Many procedures were examined for this purpose may be by selecting resistant breeds, using anti cold stress agents or feed additives. Chicken phenotype involves body shape, feather model, eyes color and comb type. The Crest phenotype is characterized by a bunch of outspread feathers upper the head (Wang et al., 2012) and found in many bird species (Price, 2002). The appearance of the Crest depends on the length and shape of the cranial feathers (Wang et al., 2012).Crest is controlled by autosomal incompletely dominant gene (Fathi and Galal, 2001) and classical genetics experiments suggest that the head crest segregates as a simple Mendelian recessive trait(Wang et al., 2012).Feather mass of crest phenotype chickens increased by about 12\% (Galal, 2003) and crested chickens may be a good layer(Thomas et al., 2010).Dandarawi chicken was developed in Upper Egypt and could be simply sexed by feather color. Many authors studied crest in Dandarawi chicken where Fathi et al. (2000) reported that crest gene in Dandarawi chicken remarkably improved the abnormality and viability of spermatozoa.

L-arginine is an essential amino acid for poultry where they cannot synthesize it nevertheless chickens have the highest Arg requirement of all animals (Ball et al., 2007). Many molecules, including glutamate, ornithine, creatine, and nitric oxide created from Arg (Khajali and Wideman, 2010).Nitric oxide production increased in the duodenum of chronically chicks reared under chronic cold stress (Zhang et al., 2011). Arginine supplementation improves cardiovascular when birds exposed to cold stress (RuizFeria, 2009) and may reduce the undesirable effects of cold stress (AlDaraji and Salih,2012). Arginine requirements of NRC may inadequate to overcome cold stress (Khajali and Wideman, 2010) and Arg supplementation had positive effects on cold stress (Bozakova and Gerzilov, 2014). Beside Arg effects on cold stress, Arg with methionine supplementation play an important role in increasing feather weight, so it required for feather growth and feather development (Nazem et al., 2015). In general Cold stress occurs when the ambient temperature was lower than $18^{\circ} \mathrm{C}$ (Zhang et al., 2011).

Therefore the present experiment was carried out to evaluate response of crested (Cr) and non crested (cr) Dandarawi laying hens and excess Arg supplementation during winter months on productive and reproductive performance.

\section{MATERIALS AND METHODS}

This experiment was conducted during the period from 21 November to 21 February at Fayoum poultry research station. Ambient temperature and relative humidity were recorded daily and the average of high and low ambient temperature and relative humidity were summarized in Table 1.

\section{Experimental design and productive performance:}

Seventy two female from each of $\mathrm{Cr}$ and cr Dandarawi laying hens were chosen when theyreached50\% egg production percent $(\mathrm{EP} \%)$. The $\mathrm{Cr}$ and cr phenotypes were distributed randomly into 3 experimental groups; each group consists of 24 hens divided randomly into 3 replicates ( 8 hens each).Groups 1 from each type were fed control basal diet (Table 2) that satisfied recommendations of NRC (1994). Groups 2 and 3 from each 
Crest, Dandarawi, arginine, cold stress and egg production.

type fed basal diet supplemented with 2 and 4\% from L- arginine of NRC (1994) requirements respectively. Arginine supplemented by $14 \mathrm{gm} / 100 \mathrm{~kg}$ and $28 \mathrm{gm} / 100 \mathrm{~kg}$ to basal diet to perform levels $2 \%$ and $4 \%$ respectively.

Each bird was housed separately in single cage for three months. Eggs from each replicate were collected, numbered and weighed daily. Egg production percent, average egg weight and egg mass per hen per day were calculated according to Murugesan and Persia (2013). All birds fed ad libitum consequently feed consumption was recorded and feed conversion was calculated. Ten eggs from each replicate were chosen and egg quality was measured during the third month of egg production.

\section{Rectal temperature and respiration rate:}

At the end of $3^{\text {rd }}$ month of egg production two hens from each replicate were chosen randomly while, respiration rate and rectal temperature were recorded. Rectal temperature $\left({ }^{\circ} \mathrm{C}\right)$ was measured by inserting thermometers approximately 5 $\mathrm{cm}$ into rectal. Respiration rate was recorded by counting the wave cycles of breast up and down per minute. The ambient temperature was $15^{\circ} \mathrm{C}$ when these items were measured.

\section{Fertility and hatchability percentages:}

After three months of EP each replicate transferred from its single cage to floor pen to determine reproductive performance. One male from either $\mathrm{Cr}$ or cr was added to each replicate according to female phenotype. Twenty hatching eggs from each replicate within each arginine level and head feather style were selected and hatched in chick master incubator. At 7 day of incubation eggs were examined by candling to recognize infertile eggs and fertility percent was calculated. Unhatched eggs were counted and hatchability percent per total and fertile eggs were computed.

\section{Statistical analysis:}

For data analysis, general linear model "univariate model" procedure of SPSS (2007) was used. Significant scales $(\mathrm{P} \leq 0.05)$ were tested using Duncan's Multiple Range Test (Duncan, 1955).

\section{RESULTS AND DISCUSSION}

\section{Egg production (EP)}

The data presented in Table3showed that $\mathrm{EP} \%$ of Cr was significantly higher than cr during second month and for the overall period. These results agree with those of Galal and Fathi(2002) who reported that presence of $\mathrm{Cr}$ allele increased egg mass and $\mathrm{EP} \%$ by about $4.2 \%$ and $2.7 \%$ respectively compared with cr genotype. The $\mathrm{Cr}$ hens at $1^{\text {st }}$ month of EP exhibited lower EP\% and egg mass (gm) per hen per day. Later results agree with Hussein (2002) and El-Safty (2006) who observed that $\mathrm{cr}$ hens produced higher egg mass and number than $\mathrm{Cr}$ hens. The conflicting results of EP during successive months may be due to $\mathrm{Cr}$ phenotype able to tolerate cold stress than $\mathrm{cr}$ where $\mathrm{Cr}$ possesses deep feather coat than cr. This may be due to the ambient temperature during $1^{\text {st }}$ month of egg production was slightly higher than $2^{\text {nd }}$ and $3^{\text {rd }}$ months of egg production (Table, 1).

Diets supplemented with different arginine levels above (NRC 1994) recorded significantly $(\mathrm{P} \leq 0.05)$ higher $\mathrm{EP} \%$ than control diet during $2^{\text {nd }}, 3^{\text {rd }}$ month and for overall period. This result is in agreement with Youssef et al. (2015) who reported that Arg supplementation significantly increased EP\%. Increasing $\mathrm{EP} \%$ may be due to Arg stimulate secretion of luteinizing hormone (Basiouni, 2009).Moreover Arg is an essential amino acid for chickens because 


\section{S. F. Youssef et al.}

they cannot biosynthesize arginine (Allen, 1999).Crested hens that treated with $4 \%$ Arg above NRC recorded the highest egg production percent and egg mass per hen per day during the second month of egg production. This is may be due to Arg required for feather growth and feather development (Nazem et al., 2015) on the other hand crested chicken possess more feather mass(Fathi and Galal, 2001). The lowest ambient temperature was recorded in the $2^{\text {nd }}$ month of egg production (Table, 1). So feather coat may be play important role in cold condition, where Mason, et al., 2007 reported that increases in feather mass may aid in cold tolerance of birds. Hens that fed diet supplemented with $2 \%$ and $4 \%$ recorded significantly $(\mathrm{P} \leq$ $0.05)$ higher egg mass per hen per day than hens fed basal diet. This result was in full agreement with Youssef et al. (2015). This may be due to increasing EP\% that increase egg yields nevertheless control treatment recorded significantly $(\mathrm{P} \leq$ 0.05)higher average egg weight than Arg treatments for overall period.

\section{Feed intake and feed conversion}

Crested chicken consumed significantly ( $\mathrm{P}$ $\leq 0.05)$ lower diets than cr during the first month of EP (Table, 4). Excess L-Arg supplementation reduced significantly feed intake during the $1^{\text {st }}$ month of EP, but increased significantly feed intake during the $3^{\text {rd }}$ month. The conflicting results of feed intake may be due to the pattern of EP as discussed later. The results of 3rd month agree with Yang et al. (2016) who reported that Arg induced a strong reduction of feed intake.

During the first three months of EP and overall period phenotypic $\mathrm{Cr}$ improved feed conversion that becomes significant during the $2^{\text {nd }}$ month of EP. This is may be due to the birds carrying $\mathrm{Cr}$ gene may be need less energy for maintenance of body temperature compared to non-crested birds under low ambient temperatures (Galal, 2003 and El-Safty, 2006).

Crested hens that treated with $4 \%$ Arg above NRC recorded the best feed conversion ratio. This is may be due to $\mathrm{Cr}$ phenotype possesses more feather than $\mathrm{cr}$ phenotype and 4\% Arg improved feather growth. In regardless this point Leeson and Morrison (1978) reported that there was a significant correlation between feather weight and feed efficiency.

\section{Egg quality}

Most egg quality parameters were not affected significantly by $\mathrm{Cr}$ phenotype except for yolk percent and shell thickness (Table 5). Significant decrease was observed in yolk percent of egg laid by $\mathrm{Cr}$ hens but significant increase was observed in shell thickness. Results of shell thickness and shell weight percent were not harmonious. Galal and Fathi (2002) demonstrated that crest allele decreased shell percent and increased shell thickness. This led us supposed that the eggshell ultrastructure may be differ between $\mathrm{Cr}$ and $\mathrm{cr}$ therefore additional study may needed to explain this point.

Increasing dietary Arg level by $4 \%$ higher than NRC(1994) increased significantly (P $\leq$ 0.05) yolk percent and decreased significantly $(\mathrm{P} \leq 0.05)$ albumin percent. Similar results obtained by Najib and Basiouni (2004). Increasing yolk percent may be due to increasing Arg level decreased body fat content especially abdominal fat where, $\mathrm{Wu}$ et al. (2011) found that increasing Arg by $1.0 \%$ above NRC (1994) requirements decreased abdominal fat. Decreasing body fat may be increase the ability of laying hens to released fat in egg yolk.

Rectal temperature and respiration rate Crest phenotype had no significant effect on rectal temperature during cold stress 
Crest, Dandarawi, arginine, cold stress and egg production.

(Table, 6).Rectal body temperature of $\mathrm{Cr}$ phenotype during cold stress was lower by about $0.38^{\circ} \mathrm{Cthan}$ cr phenotype. This result in agreement with Galal and Fathi(2002) who reported that $\mathrm{Cr}$ recorded lower rectal temperature by about $0.26^{\circ} \mathrm{C}$ than cr. This may be due to $\mathrm{Cr}$ phenotype had higher feather mass by about $11.8 \%$ compared with cr (Fathi and Galal, 2001).Feather mass associated positively with body temperature under high ambient temperature (Deeb and Cahaner, 1999) and reduction in feather mass was associated with an increase in body temperature(Boothet al.,1993).

Crested phenotypic hens had lower significantly respiration rate than cr during chronic cold stress $\left(15^{\circ} \mathrm{C}\right)$ this may be due to increasing feather mass of $\mathrm{Cr}$ improved performance of respiratory system during growing periods. Prum (1999) reported that early feathers play function in thermal insulation.

Different Arg levels had no significant effect on respiration rate nevertheless it decreased respiration rate numerically during chronic cold stress. Decreasing numerically respiration rate by increasing Arg level during cold stress may be due to Nitric oxide synthesized from Arg (Khajali and Wideman, 2010) moreover Nitric oxide and its derivatives inhibit mitochondrial respiration (Brown, 2001).
Fertility and hatchability percentages: Insignificant improvement of fertility and hatchability percent were observed for $\mathrm{Cr}$ phenotype compared with cr phenotype (Table, 7). Fertility\%, hatchability per fertile eggs $\%$ and per total eggs $\%$ of $\mathrm{Cr}$ hens increased by 2.2, 1.8 and 3.7 respectively than cr hens. The results agree with Paul (2017) who demonstrated that $\mathrm{Cr}$ has higher hatchability percent by about 3\% than normal cr.

Supplemented Arg above NRC requirements improved fertility\%, hatchability per fertile eggs $\%$ and hatchability per total eggs $\%$. These results were in disagreement with Youssef et al. (2015) who found significant improvement of the same Arg levels on hatchability parameters. This may be due to breed difference where Dandarawi chicken was small size breed that may be need less requirement.

\section{CONCLUSION}

In conclusion $\mathrm{Cr}$ phenotype may be posses ability to overcome cold stress and able to improve productive and reproductive performance than cr phenotype. On the other hand excess Arg supplementation may be reduce side effects of cold stress during winter months. 
S. F. Youssefet al.

Table (1): average ambient temperature and relative humidity during experimental months.

\begin{tabular}{|c|c|c|c|}
\hline \multirow{2}{*}{$\begin{array}{l}\text { Month of egg } \\
\text { production }\end{array}$} & \multicolumn{2}{|c|}{ Average ambient temperature ${ }^{\circ} \mathrm{C}$} & \multirow{2}{*}{$\begin{array}{l}\text { Average relative } \\
\text { humidity } \%\end{array}$} \\
\hline & High & Low & \\
\hline First month & 19 & 14 & 51 \\
\hline Second month & 16 & 11 & 56 \\
\hline Third month & 17 & 12 & 58 \\
\hline
\end{tabular}

Table (2): Composition and calculated analysis of basal diet.

\begin{tabular}{|l|c|}
\hline Ingredients & Basal diet \\
\hline Yellow corn & 65.00 \\
Soybean meal (44\% CP) & 5.30 \\
Corn gluten (60\% CP) & 9.00 \\
Wheat bran & 12.00 \\
Di-calcium phosphate & 2.39 \\
Lime stone & 5.63 \\
Salt & 0.37 \\
Premix & 0.30 \\
DL- methionine & 0.01 \\
L-Arg & 0 \\
Total & 100 \\
\hline Calculated analysis & \\
\hline CP & 15.14 \\
ME. & 2786.49 \\
Ca & 3.45 \\
Av.P & 0.37 \\
Lys. & 0.70 \\
Met. & 0.31 \\
SAA & 0.61 \\
Na & 0.17 \\
L-Arg & .70 \\
\hline
\end{tabular}

Arginine supplemented by $14 \mathrm{gm} / 100 \mathrm{~kg}$ and $28 \mathrm{gm} / 100 \mathrm{~kg}$ to basal diet to perform levels $2 \%$ and $4 \%$ respectively.

Premix contain per $3 \mathrm{~kg}$ vit A 12000 000, vit D3 $3000000 \mathrm{IU}$, vit E 50000mg, vit K3 $3000 \mathrm{mg}$, vit B1 2000mg, vit B2 7500mg, vit B6 $3500 \mathrm{mg}$, vit B12 15mg, Pantothenic acid $12000 \mathrm{mg}$, Niacin $30000 \mathrm{mg}$, Biotin $150 \mathrm{mg}$, Folic acid $1500 \mathrm{mg}$, Choline $300 \mathrm{gm}$, Selenium $300 \mathrm{mg}$, Copper $10000 \mathrm{mg}$, Iron $40000 \mathrm{mg}$, Manganese $80000 \mathrm{mg}$, Zinc $80000 \mathrm{mg}$, Iodine $2000 \mathrm{mg}$, Cobalt $250 \mathrm{mg}$ and $\mathrm{CaCO} 3$ to $3000 \mathrm{~g}$. 
Table (3): Effect of crest phenotypes of Dandarawi laying hens, arginine levels and their interaction on egg production traits.

\begin{tabular}{|c|c|c|c|c|c|c|c|c|c|c|c|c|}
\hline \multirow{2}{*}{$\begin{array}{l}\text { Traits } \\
\text { Main } \\
\text { effects }\end{array}$} & \multicolumn{4}{|c|}{ Egg production \% } & \multicolumn{4}{|c|}{ Egg mass / hen / day (gm) } & \multicolumn{4}{|c|}{ Average egg weight (gm) } \\
\hline & $\begin{array}{c}1^{\text {st }} \\
\text { month }\end{array}$ & $\begin{array}{c}2^{\text {nd }} \\
\text { month }\end{array}$ & $\begin{array}{c}3^{\text {rd }} \\
\text { month }\end{array}$ & $\begin{array}{l}\text { Overall } \\
\text { period }\end{array}$ & $\begin{array}{c}1^{\text {st }} \\
\text { month }\end{array}$ & $\begin{array}{c}2^{\text {nd }} \\
\text { month }\end{array}$ & $\begin{array}{c}3^{\text {rd }} \\
\text { month }\end{array}$ & $\begin{array}{c}\text { Overall } \\
\text { period }\end{array}$ & $\begin{array}{c}1^{\text {st }} \\
\text { month }\end{array}$ & $\begin{array}{c}2^{\text {nd }} \\
\text { month }\end{array}$ & $\begin{array}{c}3^{\text {rd }} \\
\text { month }\end{array}$ & $\begin{array}{l}\text { Overall } \\
\text { period }\end{array}$ \\
\hline \multicolumn{13}{|l|}{ Phenotype } \\
\hline $\mathrm{cr}$ & 67.06 & $45.04^{\mathrm{b}}$ & 49.89 & $54.18^{\mathrm{b}}$ & 27.95 & $18.43^{\mathrm{b}}$ & 21.01 & $22.53^{\mathrm{b}}$ & 41.75 & $41.15^{\mathrm{b}}$ & 42.20 & $41.67^{b}$ \\
\hline $\mathrm{Cr}$ & 63.69 & $56.15^{\mathrm{a}}$ & 51.70 & $57.42^{\mathrm{a}}$ & 26.56 & $23.70^{\mathrm{a}}$ & 21.93 & $24.16^{\mathrm{a}}$ & 41.74 & $42.31^{\mathrm{a}}$ & 42.47 & $42.15^{\mathrm{a}}$ \\
\hline $\mathrm{SE} \pm$ & 2.45 & 2.50 & 2.39 & 1.57 & 1.02 & 1.05 & 1.02 & 0.66 & 0.24 & 0.56 & 0.27 & 0.15 \\
\hline Probability & N.S. & 0.002 & N.S. & 0.042 & N.S. & 0.001 & N.S. & 0.045 & N.S. & 0.002 & N.S. & 0.022 \\
\hline \multicolumn{13}{|l|}{ Arg levels } \\
\hline Zero & 66.67 & $44.35^{\mathrm{b}}$ & $37.08^{c}$ & $49.90^{\mathrm{b}}$ & 28.20 & 18.81 & $15.71^{\mathrm{c}}$ & $21.13^{\mathrm{b}}$ & 42.25 & $42.49^{\mathrm{a}}$ & 42.65 & $42.45^{\mathrm{a}}$ \\
\hline $2 \%$ & 65.18 & $52.98^{\mathrm{a}}$ & $52.38^{\mathrm{b}}$ & $57.04^{\mathrm{a}}$ & 26.78 & 21.65 & $21.97^{\mathrm{b}}$ & $23.53^{\mathrm{a}}$ & 41.41 & $40.87^{\mathrm{b}}$ & 41.98 & $41.39^{\mathrm{b}}$ \\
\hline $4 \%$ & 64.29 & $54.46^{\mathrm{a}}$ & $62.93^{\mathrm{a}}$ & $60.46^{\mathrm{a}}$ & 26.79 & 22.72 & $26.74^{\mathrm{a}}$ & $25.36^{\mathrm{a}}$ & 41.58 & $41.84^{\mathrm{a}}$ & 42.37 & $41.91^{\mathrm{c}}$ \\
\hline $\mathrm{SE} \pm$ & 3.01 & 3.07 & 2.93 & 1.92 & 1.25 & 1.28 & 1.25 & 0.81 & 0.29 & 0.32 & 0.33 & 0.18 \\
\hline Probability & N.S. & 0.045 & 0.001 & 0.001 & N.S. & N.S. & 0.001 & 0.001 & N.S. & 0.002 & N.S. & 0.001 \\
\hline \multicolumn{13}{|l|}{ Interaction } \\
\hline $\mathrm{cr} \times \operatorname{Arg} 0 \%$ & 70.83 & $35.72^{\mathrm{d}}$ & 32.65 & 47.00 & 29.92 & $14.98^{c}$ & 13.85 & 19.83 & 42.14 & $42.20^{\mathrm{a}}$ & 42.53 & $42.27^{\mathrm{ab}}$ \\
\hline $\operatorname{cr} \times \operatorname{Arg} 2 \%$ & 63.10 & $57.14^{\mathrm{ab}}$ & 51.02 & 57.35 & 25.70 & $22.70^{\mathrm{ab}}$ & 21.07 & 23.25 & 41.15 & $39.33^{\mathrm{b}}$ & 41.50 & $40.62^{c}$ \\
\hline $\mathrm{cr} \times \operatorname{Arg} 4 \%$ & 67.26 & $42.26^{\mathrm{cd}}$ & 65.99 & 58.18 & 28.22 & $17.59^{\mathrm{bc}}$ & 28.13 & 24.50 & 41.96 & $41.92^{\mathrm{a}}$ & 42.56 & $42.13^{\mathrm{ab}}$ \\
\hline $\mathrm{Cr} \times \mathrm{Arg} 0 \%$ & 62.50 & $52.98^{b c}$ & 41.50 & 52.80 & 26.48 & $22.64^{\mathrm{ab}}$ & 17.57 & 22.43 & 42.36 & $42.77^{\mathrm{a}}$ & 42.77 & $42.62^{\mathrm{a}}$ \\
\hline $\mathrm{Cr} \times \mathrm{Arg} 2 \%$ & 67.26 & $48.81^{\mathrm{bc}}$ & 53.74 & 56.73 & 27.86 & $20.61^{\mathrm{b}}$ & 22.87 & 23.82 & 41.66 & $42.41^{\mathrm{a}}$ & 42.46 & $42.15^{\mathrm{ab}}$ \\
\hline $\mathrm{Cr} \times \mathrm{Arg} 4 \%$ & 61.31 & $66.67^{\mathrm{a}}$ & 59.87 & 62.73 & 25.35 & $27.85^{\mathrm{a}}$ & 25.36 & 26.22 & 41.20 & $41.75^{\mathrm{a}}$ & 42.19 & $41.69^{b}$ \\
\hline $\mathrm{SE} \pm$ & 4.25 & 4.34 & 4.14 & 2.71 & 1.76 & 1.81 & 1.77 & 1.14 & 0.41 & 0.45 & 0.47 & 0.26 \\
\hline Probability & N.S. & 0.001 & N.S. & N.S. & N.S. & 0.002 & N.S. & N.S. & N.S. & 0.001 & N.S. & 0.001 \\
\hline
\end{tabular}

Cr: crested Dandarawi chicken cr: non-crested Dandarawi chicken

$* a, \mathrm{~b}, \ldots=$ Means in the same column within each main effect with different superscripts, differ significantly $(\mathrm{P}<0.05)$;

N.S. $=$ Not Significant $(\mathrm{P}>0.05)$. 
Table (4): Effect of crest phenotypes of Dandarawi laying hens, arginine levels and their interaction on feed intake and feed conversion.

\begin{tabular}{|c|c|c|c|c|c|c|c|c|}
\hline \multirow{2}{*}{$\begin{array}{l}\text { Traits } \\
\text { Main effects }\end{array}$} & \multicolumn{4}{|c|}{ Feed intake (hen/day) gm } & \multicolumn{4}{|c|}{ Feed conversion (gm feed/gm egg) } \\
\hline & $\begin{array}{c}1^{\text {st }} \\
\text { month }\end{array}$ & $\begin{array}{c}2^{\text {nd }} \\
\text { month }\end{array}$ & $\begin{array}{c}3^{\text {rd }} \\
\text { month }\end{array}$ & $\begin{array}{c}\text { Overall } \\
\text { period }\end{array}$ & $\begin{array}{c}1^{\text {st }} \\
\text { month }\end{array}$ & $\begin{array}{c}2^{\text {nd }} \\
\text { month }\end{array}$ & $\begin{array}{l}3^{\text {rd }} \\
\text { month }\end{array}$ & $\begin{array}{c}\text { Overall } \\
\text { period }\end{array}$ \\
\hline \multicolumn{5}{|l|}{ Phenotype } & & & & \\
\hline cr & $86.89^{\mathrm{a}}$ & 76.56 & 78.00 & 80.48 & 3.16 & $4.46^{\mathrm{a}}$ & 4.06 & $3.89^{\mathrm{a}}$ \\
\hline $\mathrm{Cr}$ & $82.56^{\mathrm{b}}$ & 78.56 & 80.78 & 80.63 & 3.12 & $3.40^{\mathrm{b}}$ & 3.84 & $3.45^{\mathrm{b}}$ \\
\hline $\mathrm{SE} \pm$ & 1.44 & 2.34 & 1.93 & 1.45 & 0.10 & 0.28 & 0.24 & 0.16 \\
\hline Probability & 0.05 & N.S. & N.S. & N.S. & N.S. & 0.02 & N.S. & 0.045 \\
\hline \multicolumn{5}{|l|}{ Arg levels } & & & & \\
\hline Zero & $88.67^{\mathrm{a}}$ & 73.50 & $73.33^{\mathrm{c}}$ & 78.50 & 3.16 & 4.14 & $4.83^{\mathrm{a}}$ & $4.04^{\mathrm{a}}$ \\
\hline $2 \%$ & $83.50^{\mathrm{b}}$ & 81.33 & $78.83^{\mathrm{b}}$ & 81.22 & 3.18 & 3.91 & $3.73^{\mathrm{b}}$ & $3.61^{\mathrm{ab}}$ \\
\hline $4 \%$ & $82.00^{\mathrm{b}}$ & 77.83 & $86.00^{\mathrm{a}}$ & 81.94 & 3.07 & 3.75 & $3.28^{\mathrm{b}}$ & $3.37^{\mathrm{b}}$ \\
\hline $\mathrm{SE} \pm$ & 1.76 & 2.87 & 2.37 & 1.77 & 0.12 & 0.34 & 0.29 & 0.20 \\
\hline Probability & 0.04 & N.S. & 0.009 & N.S. & N.S. & N.S. & 0.007 & 0.049 \\
\hline \multicolumn{5}{|l|}{ Interaction } & & & & \\
\hline cr $\times \operatorname{Arg} 0 \%$ & 93.67 & 71.00 & 71.67 & 78.78 & 3.14 & 4.87 & 5.32 & 4.44 \\
\hline $\mathrm{cr} \times \operatorname{Arg} 2 \%$ & 83.67 & 83.67 & 77.67 & 81.67 & 3.37 & 3.92 & 3.83 & 3.71 \\
\hline cr $\times \operatorname{Arg} 4 \%$ & 83.33 & 75.00 & 84.67 & 81.00 & 2.96 & 4.59 & 3.01 & 3.52 \\
\hline $\mathrm{Cr} \times \operatorname{Arg} 0 \%$ & 83.67 & 76.00 & 75.00 & 78.22 & 3.18 & 3.40 & 4.34 & 3.64 \\
\hline $\mathrm{Cr} \times \operatorname{Arg} 2 \%$ & 83.33 & 79.00 & 80.00 & 80.78 & 300 & 3.89 & 3.63 & 3.51 \\
\hline $\mathrm{Cr} \times \operatorname{Arg} 4 \%$ & 80.67 & 80.67 & 87.33 & 82.89 & 3.18 & 2.90 & 3.55 & 3.21 \\
\hline $\mathrm{SE} \pm$ & 2.49 & 4.05 & 3.34 & 2.50 & 0.18 & 0.48 & 0.41 & 0.28 \\
\hline Probability & N.S. & N.S. & N.S. & N.S. & N.S. & N.S. & N.S. & N.S. \\
\hline
\end{tabular}

$* a, b, \ldots=$ Means in the same column within each main effect with different superscripts, differ significantly $(\mathrm{P}<0.05)$; N.S. $=$ Not Significant $(\mathrm{P}>0.05)$. 
Table (5): Effect of crest phenotypes of Dandarawi laying hens, arginine levels and their interaction on egg quality parameters.

\begin{tabular}{|c|c|c|c|c|c|c|c|c|c|}
\hline $\begin{array}{l}\text { Traits } \\
\text { Main effects }\end{array}$ & $\begin{array}{l}\text { Shape } \\
\text { index }\end{array}$ & $\begin{array}{l}\text { Yolk } \\
\text { index }\end{array}$ & $\begin{array}{c}\text { Haugh } \\
\text { unit }\end{array}$ & Yolk\% & Albumin \% & Shell\% & $\begin{array}{c}\text { Shell } \\
\text { thickness }\end{array}$ & Sa & $\begin{array}{l}\text { Sw/ } \\
\text { Sa }\end{array}$ \\
\hline \multicolumn{10}{|l|}{ Phenotype } \\
\hline cr & 77.29 & 41.67 & 94.70 & $32.75^{\mathrm{a}}$ & 56.78 & 11.58 & $39.02^{b}$ & 55.59 & 88.15 \\
\hline $\mathrm{Cr}$ & 77.38 & 41.88 & 95.06 & $32.10^{\mathrm{b}}$ & 57.04 & 10.87 & $40.65^{\mathrm{a}}$ & 56.12 & 82.84 \\
\hline $\mathrm{SE} \pm$ & 0.53 & 0.35 & 0.62 & 0.23 & 0.25 & 0.80 & 0.39 & 0.31 & 6.01 \\
\hline Probability & N.S. & N.S. & N.S. & 0.046 & N.S. & N.S. & 0.003 & N.S. & N.S. \\
\hline \multicolumn{10}{|l|}{ Arg levels } \\
\hline Zero & 77.18 & 40.99 & 94.28 & $32.19^{b}$ & $57.06^{\mathrm{a}}$ & 10.75 & 40.76 & 56.13 & 81.66 \\
\hline $2 \%$ & 77.06 & 42.17 & 96.12 & $31.88^{\mathrm{b}}$ & $57.55^{\mathrm{a}}$ & 10.56 & 39.57 & 55.97 & 81.06 \\
\hline $4 \%$ & 77.76 & 42.18 & 94.24 & $33.20^{\mathrm{a}}$ & $56.10^{\mathrm{b}}$ & 12.36 & 39.17 & 55.47 & 93.77 \\
\hline $\mathbf{S E} \pm$ & 0.65 & 0.42 & 0.76 & 0.28 & 0.31 & 0.98 & 0.47 & 0.38 & 6.47 \\
\hline Probability & N.S. & N.S. & N.S. & 0.003 & 0.003 & N.S. & N.S. & N.S. & N.S. \\
\hline \multicolumn{10}{|l|}{ Interaction } \\
\hline cr $\times \operatorname{Arg} 0 \%$ & 77.11 & 40.87 & 94.56 & 32.56 & 57.10 & 10.35 & 40.31 & 56.60 & 78.77 \\
\hline $\operatorname{cr} \times \operatorname{Arg} 2 \%$ & 76.44 & 41.99 & 95.52 & 31.99 & 57.53 & 10.48 & 38.94 & 55.69 & 81.00 \\
\hline cr $\times \operatorname{Arg} 4 \%$ & 78.31 & 42.16 & 94.01 & 33.70 & 55.70 & 13.92 & 37.80 & 54.48 & 104.68 \\
\hline $\mathrm{Cr} \times \operatorname{Arg~0\% }$ & 77.25 & 41.10 & 94.00 & 31.81 & 57.03 & 11.16 & 41.21 & 55.66 & 84.55 \\
\hline $\mathrm{Cr} \times \operatorname{Arg} 2 \%$ & 77.68 & 42.34 & 96.72 & 31.78 & 57.57 & 10.65 & 40.19 & 56.24 & 81.13 \\
\hline $\operatorname{Cr} \times \operatorname{Arg} 4 \%$ & 77.22 & 42.20 & 94.47 & 32.70 & 56.50 & 10.80 & 40.53 & 56.46 & 82.86 \\
\hline $\mathrm{SE} \pm$ & 0.91 & 0.60 & 1.07 & 0.39 & 0.43 & 1.39 & 0.67 & 0.54 & 10.57 \\
\hline Probability & N.S. & N.S. & N.S. & N.S. & N.S. & N.S. & N.S. & N.S. & N.S. \\
\hline
\end{tabular}

Sa: Surface area - Sw/Sa: shell weight/unit of surface area -Cr: crested Dandarawi chicken -cr: non-crested Dandarawi chicken $* a, b, \ldots=$ Means in the same column within each main effect with different superscripts, differ significantly $(\mathrm{P}<0.05)$;

N.S. $=$ Not Significant $(\mathrm{P}>0.05)$. 
S. F. Youssef et al.

Table (6): Effect of crest phenotypes of Dandarawi laying hens, arginine levels and their interaction on body temperature and respiration rate during cold stress $\left(15^{\circ} \mathrm{C}\right)$.

\begin{tabular}{|l|c|c|}
\hline Traits & BT & R.R. \\
\hline Main effects & \multicolumn{2}{|c|}{} \\
\hline Phenotype & 42.18 & $37.67^{\mathrm{a}}$ \\
\hline cr & 41.80 & $29.00^{\mathrm{b}}$ \\
Cr & 0.160 & 1.054 \\
SE \pm & N.S. & 0.001 \\
Probability & \multicolumn{2}{|l|}{} \\
\hline Arg levels & 42.08 & 34.00 \\
\hline Zero & 41.75 & 33.50 \\
2\% & 42.15 & 32.50 \\
$4 \%$ & 0.196 & 1.291 \\
SE \pm & N.S. & N.S. \\
Probability & \multicolumn{2}{|c|}{} \\
\hline Interaction & 42.15 & 37.00 \\
\hline cr $\times$ Arg 0\% & 42.10 & 38.00 \\
cr $\times$ Arg 2\% & 42.30 & 38.00 \\
cr $\times$ Arg 4\% & 42.00 & 31.00 \\
Cr $\times$ Arg 0\% & 41.40 & 29.00 \\
Cr $\times$ Arg 2\% & 42.00 & 27.00 \\
Cr $\times$ Arg 4\% & 0.278 & 1.829 \\
Probability & N.S. & N.S. \\
SE \pm &
\end{tabular}

BT: boy temperature $\quad$ R.R.: respiration rate

Cr: crested Dandarawi chicken cr: non-crested Dandarawi chicken

$* a, b, \ldots=$ Means in the same column within each main effect with different superscripts, differ significantly $(\mathrm{P}<0.05) ;$ N.S. $=$ Not Significant $(\mathrm{P}>0.05)$. 
Table (7) : Effect of phenotype of Dandarawi chickens, arginine levels and their interaction on fertility and hatchability percent.

\begin{tabular}{|l|c|c|c|}
\hline $\begin{array}{l}\text { Traits } \\
\text { Main effects }\end{array}$ & Fertility\% & $\begin{array}{c}\text { Hatch. } \\
\text { F. eggs\% }\end{array}$ & $\begin{array}{c}\text { Hatch. } \\
\text { T. eggs\% }\end{array}$ \\
\hline Phenotype & \multicolumn{3}{|c|}{} \\
\hline cr & 88.15 & 88.79 & 78.52 \\
Cr & 90.37 & 90.58 & 82.22 \\
SE \pm & 1.960 & 2.582 & 3.629 \\
Probability & N.S. & N.S. & N.S. \\
\hline Arg levels & \multicolumn{3}{|c|}{} \\
\hline Zero & 85.56 & 84.28 & 72.22 \\
2\% & 88.89 & 91.09 & 81.11 \\
$4 \%$ & 93.33 & 93.68 & 87.78 \\
SE \pm & 2.400 & 3.162 & 4.444 \\
Probability & N.S. N.S. & N.S. \\
\hline Interaction & \multicolumn{3}{|c|}{} \\
\hline cr $\times$ Arg 0\% & 84.44 & 84.19 & 71.11 \\
cr $\times$ Arg 2\% & 86.67 & 87.12 & 75.56 \\
cr $\times$ Arg 4\% & 93.33 & 95.06 & 88.89 \\
Cr $\times$ Arg 0\% & 86.67 & 84.37 & 73.33 \\
Cr $\times$ Arg 2\% & 91.11 & 95.06 & 86.67 \\
Cr $\times$ Arg 4\% & 93.33 & 92.31 & 86.67 \\
SE. & 3.395 & 4.472 & 6.285 \\
Probability & N.S. & N.S. & N.S. \\
\hline
\end{tabular}

Cr: crested Dandarawi chicken cr: non-crested Dandarawi chicken

Hatch.F. eggs\%: hatchability per fertile eggs Hatch.T. eggs\%: hatchability per total eggs $\mathrm{a}, \mathrm{b}, \ldots=$ Means in the same column with within each main effect different superscripts, differ significantly $(\mathrm{P}<0.05)$; N.S. $=$ Not Significant $(\mathrm{P}>0.05)$. 


\section{REFERENCES}

Al-Daraji, H. J.; and Salih, A.M. 2012. Effect of dietary L-arginine on carcass traits of broilers. Res. Opin. Anim. Vet. Sci., 2: 40-44.

Allen, P. C. 1999. Effects of daily oral doses of L-arginine on coccidiosis infections in chickens. Poult. Sci. 78:1506-1509.

Ball, R. O.; Urschel, K. L.; and Pencharz, P. B. 2007. Nutritional consequences of interspecies differences in arginine and lysine metabolism. Journal of Nutrition. 137: 1626-1641.

Basiouni, G. F. 2009. The Effect of feeding an extra amounts of arginine to local Saudi hens on luteinizing hormone secretion. J. Biolog. Sci., 9: 617-620.

Booth, D. T.; Clayton, D. H.; and Block, B.A.1993. Experimental demonstration of the energetic cost of parasitism in free-ranging hosts. Proceedings of the Royal Society of London B: Biolog. Sci. 253: 125-129.

Bozakova, N. and Gerzilov, V., 2014. Opportunities for the welfare improvement of laying hens under semi-open rearing during the cold period with arginine and vitamin $\mathrm{C}$ supplementation. Türk Tarım ve Doğa Bilimleri .6: 793-798.

Brown,G.C.2001.Regulation of mitochondrial respiration by nitric oxide inhibition of cytochrome $\mathrm{c}$ oxidase. Biochimica et Biophysica Acta-Bioenergetics. 1504:46-57.

Deeb, N. A. D. E.; and Cahaner, A. V. I. G. 1999. The effects of naked neck genotypes, ambient temperature, and feeding status and their interactions on body temperature and performance of broilers. Poult. Sci. 78:1341-1346.
Duncan, D. B. 1955. Multiple range and multiple F tests. Biomet. 11: 1-42.

El-Safty, S. A. 2006. Influence of naked neck, frizzle, crest genes and their triple segregation on productivity of layer chickens under hot environmental conditions. Egypt. Poult. Sci. 26: 12531267.

Fathi, M. M.; and Galal, A. 2001. Assessing the combined effects of naked neck and crest genes on growth performance and meat yield of chicken under high ambient temperature. Egypt. Poult. Sci. 21 : 319-338.

Fathi, M. M.; El-Wardany, I. E.; and El-Hommosany, Y. M. 2000. Genetic differences in adult males of some Egyptian local breeds of chicken fed different dietary protein levels. Egypt. Poult. Sci. 20: 271-284.

GalalA., 2003. Influence of naked neck, frizzle and crest genes on body weight and carcass measurements of male chickens under low ambient temperatures. Egypt. Poult. Sci. 23 :183-199.

Galal, A.; and M. M. Fathi 2002. Introducing crest gene to enhance productive performance of naked neck chickens under moderate ambient temperature. Egypt. Poult. Sci. 22: 611628.

Hussein, S. H. 2002. Effect of naked neck gene on laying performance in some Egyptian local breeds of chickens. Sciences Conference, Taez, Yemen Republic.1-12.

Khajali, F.; and Wideman, R. F. 2010. Dietary arginine: Metabolic, environmental, immunological and physiological interrelationships. World's Poult. Sci. J. 66: 751-766.

Leeson, S.; and Morrison, W.D., 1978. Effect of feather cover on feed 
efficiency in laying birds. Poult. Sci. 57:1094-1096.

Mason, D. D.; Barboza, P. S.; and Ward, D. H., 2007. Mass dynamics of wintering Pacific black brant: body, adipose tissue, organ, and muscle masses vary with location. Canadian Journal of Zoology, 85: 728-736.

Murugesan, G. R.; and M. E. Persia 2013. "Validation of the effects of small differences in dietary metabolizable energy and feed restriction in first-cycle laying hens." Poult. Sci. 92: 1238-1243.

Najib, H.; and Basiouni G. D. 2004. Determination of the nutritional requirements of the Local Saudi chickens. 1. Effect of Arginine inclusion, in excess of the leghorn requirement, on performance of the Local Saudi chickens. Scientific Journal of King Faisal University Basic and Applied Sci. 5:121-144.

Nazem, M. N.; Amanollahi, R.; Tavakoli, H.; and Mansouri, F 2015. Effect of in ovo injected methionine on feather follicle formation and its growth in the chicken embryo. Anatomical Sciences Journal. May 15:83-88.

NRC, National Research Council, 1994. Nutrient Requirements of Poultry. 9th revised edition. National Academy Press. Washington, D.C., USA.

Paul, M 2017. Qualitative mutant genes within the local chicken population in Ghana: their influence on growth performance and egg production. College of agriculture and natural resources. Kwame university. Kumasi. Msc.
Price, T. D. 2002. Domesticated birds as a model for the genetics of speciation by sexual selection. In Genetics of Mate Choice: From Sexual Selection to Sexual Isolation (pp. 311-327). Springer Netherlands.

Prum, R. O. 1999. Development and evolutionary origin of feathers. The Journal of experimental zoology. 285: 291-306.

Ruiz-Feria, C. A. 2009. Concurrent supplementation of arginine, vitamin E, and vitamin $\mathrm{C}$ improve cardiopulmonary performance in broilers chickens. Poult. Sci. 88:526535.

SPSS., 2007. SPSS Users Guide Statistics. Version 16. Copyright SPSS Inc., USA.

Thomas, M. M.; Muhuyi, W. B.; and Kahi, A. K. 2010. Influence of major genes for crested-head, frizzle-feather and naked-neck on body weights and growth patterns of indigenous chickens reared intensively in Kenya. Trop. Anim. Health. Prod. 42:173-183.

Wang, Y.; Gao, Y.; Imsland, F.; Gu, X.; Feng, C.; Liu, R.; Song, C.; TixierBoichard, M.; Gourichon, D.; Li, Q.; and Chen, K. W. 2012. The crest phenotype in chicken is associated with ectopic expression of $\mathrm{HOXC} 8$ in cranial skin. PLoS One, 7(4), p.e34012.

Wu, L. Y.; Fang, Y. J.; and Guo, X. Y. 2011. Dietary L-arginine supplementation beneficially regulates body fat deposition of meat-type ducks. Br. Poult. Sci. 52: 221-226.

Yang, H.; Ju, X.; Wang, Z.; Yang, Z.; Lu, J.; and Wang W. 2016. Effects of arginine supplementation on organ development, egg quality, serum biochemical 
parameters, and immune status of laying hens. Revista Brasileira de Ciência Avícola 18: 181-186.

Youssef, S. F.; Shaban, S. A. M.; and Inas, I. I., 2015. Effect of 1-arginine supplementation on productive, reproductive performance, immune response and gene expression in two local chicken strains: 1 -egg production, reproduction performance and immune response. Egypt. Poult. Sci. 35: 573590.

Zhang, Z. W.; Lv, Z. H.; Li, J. L.; Li, S.; Xu, S. W. ; and Wang X. L. 2011. "Effects of cold stress on nitric oxide in duodenum of chicks." Poult. sci. 90: 1555-15

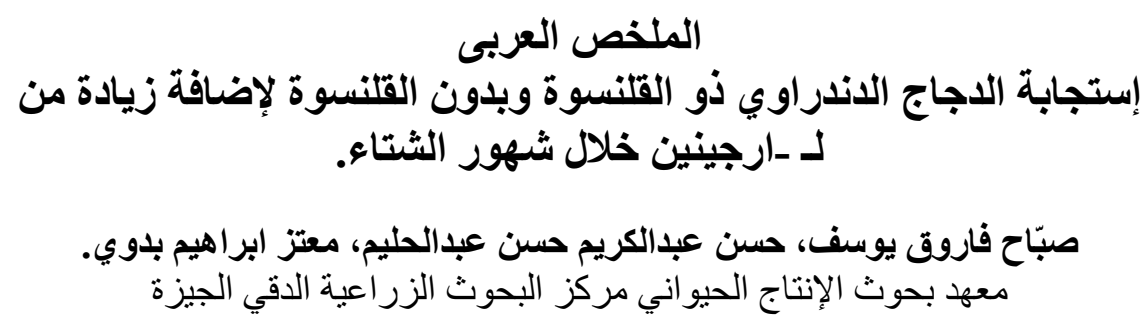

اجريت هذه التجربة خلال فصل الثتاء لتقدير استجابة خطين مختلفين ظاهرياً من دجاج الدندر اوي ذو القلنسوة وبدون

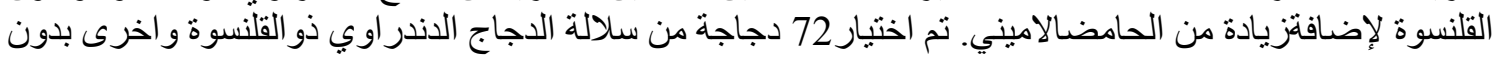

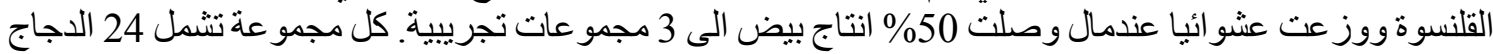

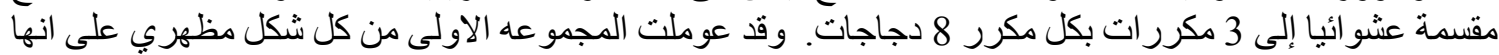

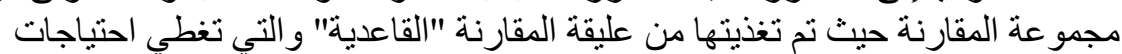

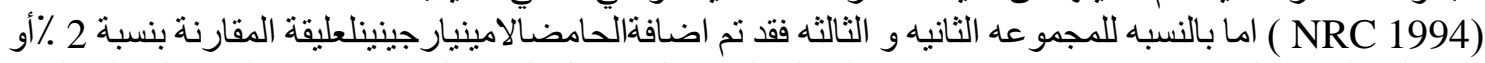

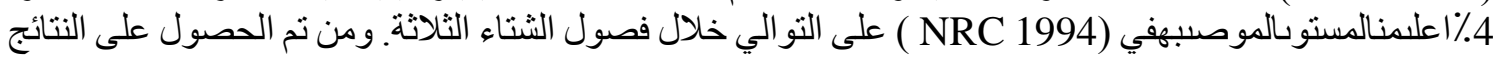

اعطى دجاج الدندراوي ذو القلنسوة نسبة إنتاج البيض مئويةاعلى معنوياً مقابل دجاج الدندر اوي بدون القلنسوة خلال

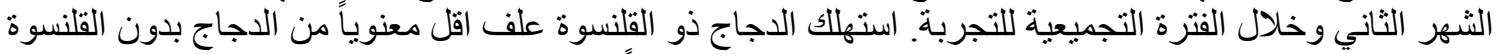

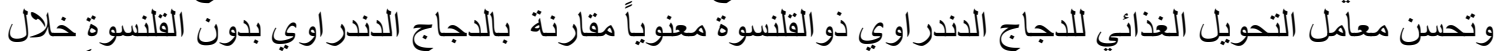

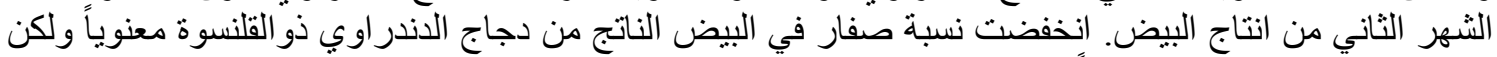

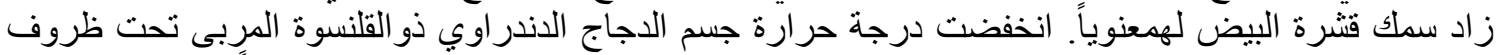

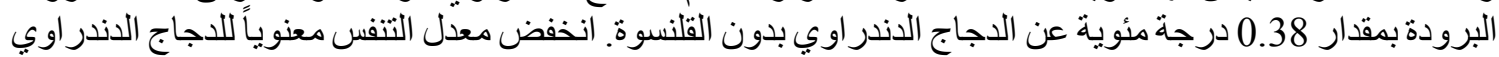

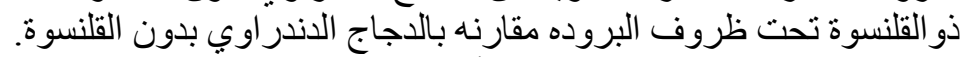

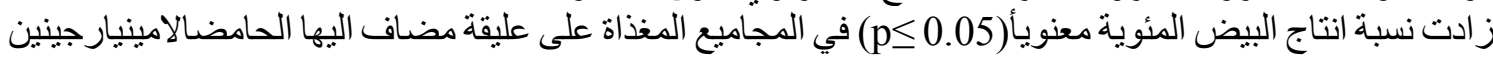

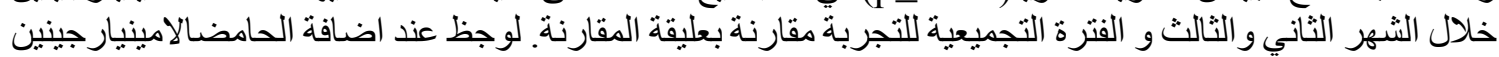

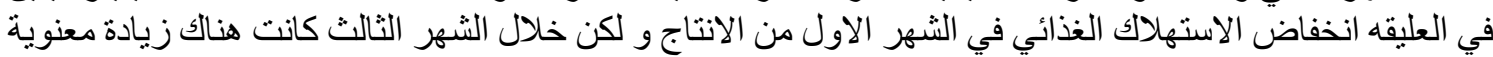

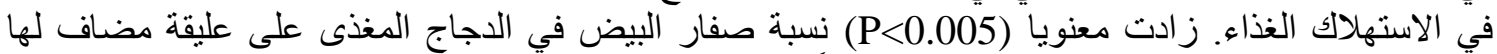

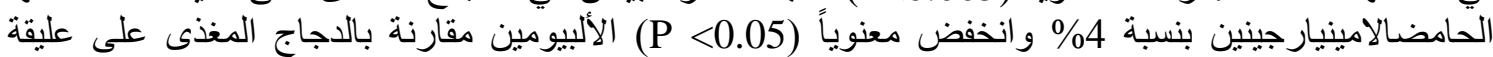

